(C) [2005] IEEE. Reprinted, with permission, from [Tony Huang and Ananda Sanagavarapu Mohan, A hybrid boundary condition for robust particle swarm optimization, Antennas and Wireless Propagation Letters, IEEE (Volume:4 ), 20 June 2005]. This material is posted here with permission of the IEEE. Such permission of the IEEE does not in any way imply IEEE endorsement of any of the University of Technology, Sydney's products or services. Internal or personal use of this material is permitted. However, permission to reprint/republish this material for advertising or promotional purposes or for creating new collective works for resale or redistribution must be obtained from the IEEE by writing to pubs-permissions@ieee.org. By choosing to view this document, you agree to all provisions of the copyright laws protecting it 


\section{A HYBRID BOUNDARY CONDITION FOR ROBUST PARTICLE SWARM OPTIMIZATION}

Tony Huang, Student Member, IEEE, and Ananda Sanagavarapu Mohan, Member, IEEE

Manuscript received October 29, 2004. This work is supported by the Australian Research Council (ARC) through a Discovery Project Grant.

The authors are with the Microwave and Wireless Technology Research Laboratory, Information and Communication Group, Faculty of Engineering, University of Technology, Sydney, NSW 2007, Australia. (email: thuang@eng.uts.edu.au; ananda@eng.uts.edu.au). 
Abstract - The particle swarm optimization (PSO) technique is a powerful stochastic evolutionary algorithm that can be used to find the global optimum solution in a complex search space. However, it has been observed that there is a great variation in its performance due to the dimensionality of the problem and the location of the global optimum with respect to the boundaries of the search space. The present paper attempts to resolve this problem by proposing a simple hybrid "damping" boundary condition that combines the characteristics offered by the existing "absorbing" and "reflecting" boundaries. Simulation results on microwave image reconstruction have shown that with the proposed "damping" boundary condition, a much robust and consistent optimization performance can be obtained for PSO regardless of the dimensionality and location of the global optimum solution.

Index Terms - Convergence of numerical methods, evolutionary computation, microwave image reconstruction, optimization methods, particle swarm. 


\section{INTRODUCTION}

The Particle Swarm Optimization (PSO) technique is a stochastic optimization technique first proposed by Eberhart and Kennedy [1], [2] and introduced to the antenna engineering community recently by Robinson and Rahmat-Samii [3].

However, like any other stochastic optimization technique, PSO suffers from the "curse of dimensionality" which means that its performance would deteriorate as the dimensionality of the problem increases [4]. Also the PSO performance will vary depending on the distance between the optimum solution and the boundary of the search space. Such a variation in the performance could represent a potential problem for using PSO effectively to solve high dimensional optimization problems in which the location of the global optimum with respect to the search space boundary is unknown. A typical example of this type of problem arises in the process of microwave image reconstruction. In such a case, the investigation domain of the problem is usually partitioned into many sub-volumes, hence high dimensionality, and the known background medium is generally used to determine the boundary of the search space before the entire inverse scattering problem is modeled as a nonlinear and ill-posed optimization problem [5].

In this paper, we propose a simple hybrid boundary condition that can be used to obtain a robust and consistent PSO performance for high dimensional optimization problems regardless of where the global optimum is located in the search space. In Section II, the PSO technique is briefly introduced along with the description of the proposed damping boundary condition. Section III presents some simulation results to highlight the robustness and consistency offered by the proposed boundary condition. The comparison of PSO performance between different boundary conditions for solving a microwave image reconstruction problem is presented in Section IV, and finally the conclusions of this paper are given in Section V.

\section{Damping Boundary Condition For Particle Swarm Opimization}

In PSO, each particle represents an alternative solution in the multi-dimensional search space. Thus these 
particles are multi-dimensional vectors whose trajectories are updated based on the velocity defined by its previous best success, $p_{\text {best }}$, and the best success achieved by the best particle in the swarm, $g_{\text {best }}$. Some useful modifications viz., use of inertia weight and constriction factors has made the original implementation of the technique very efficient [6-9].

The velocity and position of the particles are updated based on the equations below [7]:

$$
\begin{gathered}
v_{i, n}(t+1)=w v_{i, n}(t)+c_{1} r_{1}\left[p_{\text {best }}-x_{i, n}(t)\right]+c_{2} r_{2}\left[g_{\text {best }}-x_{i, n}(t)\right] \\
x_{i}(t+1)=x_{i}(t)+v_{i}(t+1)
\end{gathered}
$$

where $x_{i, n}(t)$ and $v_{i, n}(t)$ are the current location and velocity vector of the $i^{\text {th }}$ particle in its $n^{\text {th }}$ dimension. $w$ is the inertia weight used to control global exploration and local exploitation of the particles, and is usually varied linearly from 0.9 to 0.4 in a decreasing order throughout the simulation. $c_{1}$ and $c_{2}$ are the acceleration constants that act as weights to provide the relative pull for each particle towards $p_{\text {best }}$ and $g_{\text {best }}$ positions. $r_{1}$ and $r_{2}$ are two uniformly distributed random variables in the range [0,1] to provide a stochastic variation in the relative pull towards $p_{\text {best }}$ and $g_{\text {best }}$.

In most cases, a parameter $V_{\text {max }}$ that acts as an upper limit for the achievable velocity of the particles is also used to control the ability of the particles to search and be confined within the search space. However, it has been noted in [3] that the particles may still occasionally fly to a position beyond the defined search space, and hence produce an invalid solution. To solve this problem, the authors in [3] have suggested enclosing the search space with three different hypothetical boundaries each with its own boundary condition viz., absorbing walls, reflecting walls and invisible walls. The feature of each boundary condition is illustrated in Figs. 1(a)-(c). While in some cases the choice of invisible walls makes PSO to perform slightly better than the other two boundary conditions [3], in general, the PSO performance under any of these three boundary conditions will vary significantly depending on the problem dimensionality and the location of the global optimum with respect to the search space boundary. This is the main problem that is faced by PSO since it is difficult to know a priori as to which boundary condition suits a given optimization problem. 
In many practical optimization problems, the dimensionality and the location of the global optimum is usually difficult to know a priori. It is therefore desirable to have a single boundary condition that can offer a robust and consistent performance for the PSO technique regardless of the problem dimensionality and the location of the global optimum. With this aim, here we propose a novel hybrid "damping" boundary condition that combines features of both the characteristics offered by the absorbing and reflecting walls. With this proposed damping boundary condition, whenever a particle tries to escape the search space in any one of the dimensions, part of the velocity in that dimension is absorbed by the boundary and the particle is then reflected back to the search space with a damped velocity along with a reversal of sign, as shown in Fig. 1(d). This is can be implemented in a very straightforward manner. We first start by performing the same procedure as for the reflecting boundary where the magnitude and the sign of the velocity for the reflected particle are determined. The velocity is then multiplied by a damping factor, $\Delta d$, which is a random variable uniformly distributed between $[0,1]$ to create the damping effect. Since $\Delta d$ is a uniformly distributed random variable between $[0,1]$, the behavior of the proposed damping boundary will lie in between the performances of the absorbing and reflecting boundaries. It will act as the absorbing or reflecting boundary depending on the value of $\Delta d$ equal to zero or one respectively. In terms of equations, the updated velocity of the dampened particle can be expressed as

$$
v_{i, n}(t+1)=\Delta d \cdot v_{i, n, r e f}(t+1)
$$

where $v_{i, n, r e f}(t+1)$ is the velocity of the reflected particle as if the reflecting boundary were imposed at the boundary of the search space. Comparisons of the PSO performance between the proposed damping and other existing boundary conditions are presented in the next section.

\section{Simulation Results}

To compare the PSO performance for different boundary conditions, two standard test functions, viz., Rastigrin and Rosenbrock functions were employed [3]. The two functions are defined as

Rastigrin function 


$$
f_{1}(x)=\sum_{n=1}^{N}\left[x_{n}^{2}-10 \cos \left(2 \pi x_{n}\right)+10\right]
$$

Rosenbrock function

$$
f_{2}(x)=\sum_{n=1}^{N-1}\left[100\left(x_{n+1}-x_{n}^{2}\right)^{2}+\left(x_{n}-1\right)^{2}\right]
$$

where $N$ is the dimension of the test function. Note that the global minimum for both functions is equal to zero, where $f_{1}(0)=0$ and $f_{2}(1)=0$.

In our investigation, we have used two types of search spaces whose size in terms of Euclidean distance between the minimum and maximum boundaries are the same, but the search range in each dimension is different. The search range of the first (I) type is between $[-5,5]$ and the range for the second (II) type is between $[0,10]$. Here, we know that for Rastigrin function, when type I search space is used, the global optimum is located at the middle and when type II search space is used, the global optimum lies on the boundary respectively. Similarly, for Rosenbrock function, the global optimum is located close to the middle and to the boundary of the search space respectively when type I and II of the search spaces are used.

For our PSO runs, we have randomly created a set of 50 different initial populations for each type of the search space and each population consists of 30 particles. For each of the boundary condition mentioned earlier, 50 simulations were performed using the same initial population set created earlier. Each simulation consists of 200 iterations, and the results are averaged at the end of 50 simulation runs. The average fitness value versus the number of iterations is plotted in Fig. 2.

For the Rastigrin function, referring to Figs. 2(a)-(b), the global optimum is located at the middle of the search space for type I cases (left column) and is located at the boundary of the search space for type II cases (right column). It can be seen that the PSO performance obtained by each of the existing boundary condition is affected by two factors viz. (i) the increase in the dimension of the test function and (ii) variation in the location of the global optimum with respect to the search space boundary. Thus it is difficult to guarantee that the best performance in all the cases based on its previous success. This is clearly 
noticeable for the case of invisible boundary when $N=30$ for both type I and II cases. On the other hand, we can see that the proposed damping boundary has performed consistently well in all the four cases. It can also be seen that the performance of damping boundary is slightly lower than the invisible and reflecting boundaries when $N=30$ for type I case. However, the overall performance offered by the damping boundary in all the four cases is itself a good testimony of its robustness and consistency in the optimization process, regardless of the dimensionality and the location of the global optimum. In our simulations, we have also noticed that our result for $N=3$ is different to the one presented in [3], which is due to the difference in distance between the global optimum to the search space boundary. We found that the invisible boundary offers superior performance as reported in [3] only when the search space is small, e.g. when the size of the search space spans $x_{n} \in[-1,1]$. This finding has also confirmed that the performance of existing boundary conditions would vary significantly based on the distance between the global optimum and the search space boundary.

Similarly, from Figs. 2(c)-(d), where Rosenbrock function is used, the performance obtained by the existing three boundary conditions again varies for different cases, but the proposed damping boundary is still very consistent regardless of the dimensionality and the location of the global optimum solution. Hence, these results confirm that even for optimizing a high dimensional test function, with unknown location of global optimum with respect to the search space, damping boundary condition offers a robust and consistent PSO performance.

\section{Microwave Imaging ExamPLE}

Here we investigate the performance of damping boundary for reconstructing the dielectric constant of a lossless dielectric scatterer. The reconstruction process is modeled as a nonlinear optimization problem and the final image is computed iteratively. At each iterative process, the measured scattered field is compared with the scattered field computed from the numerical model of the test object, and electromagnetic profiles

of the model is then progressively adjusted by minimizing the error between the two sets of data. Hence, in 
this case, the objective function $(O F)$ can be defined as the normalized root mean square error (RMSE) between the measured and computed values of the scattered fields, i.e.

$$
O F=\sqrt{\frac{1}{M} \sum_{m=1}^{M} \frac{\left|E_{\text {meas }}^{s}\left(\rho_{m}, \phi_{m}, z_{m}\right)-E_{\text {comp }}^{s}\left(\rho_{m}, \phi_{m}, z_{m}\right)\right|^{2}}{\left|E_{\text {meas }}^{s}\left(\rho_{m}, \phi_{m}, z_{m}\right)\right|^{2}}}
$$

where $M$ is the total number of receivers used in the imaging system and $E_{\text {meas }}^{s}\left(\rho_{m}, \phi_{m}, z_{m}\right)$, $E_{\text {comp }}^{s}\left(\rho_{m}, \phi_{m}, z_{m}\right)$ are the measured and computed values of the scattered field at location $\left(\rho_{m}, \phi_{m}, z_{m}\right)$, respectively.

To demonstrate the effectiveness of the proposed damping boundary against other existing boundary conditions for microwave imaging, we have considered an example where a lossless homogeneous dielectric scatterer of $\varepsilon_{r}=6$ is located inside a cubic free space investigation domain of side length $\lambda_{0}$ [5]. The investigation domain is virtually partitioned into 27 equal sized sub-cells and only one of them houses the dielectric scatterer, as shown in Fig. 3. Since the goal here is to reconstruct the value of $\varepsilon_{r}$ for the dielectric scatterer without knowing its location within the investigation domain, we will need to reconstruct the value of $\varepsilon_{r}$ for each cell and thus the dimensionality of this reconstruction problem is equal to 27 .

For the reconstruction process, we have assumed that an incident plane wave at $2.45 \mathrm{GHz}$, is propagating along the $z$-axis, with a $y$-polarized electric field vector. We have also assumed that we know a priori that the investigation domain is mainly free space, and the dielectric constant $\varepsilon_{r}$ for the scatterer is less than or equal to 7 . With this a priori information we can define the search space for each cell as $\varepsilon_{r} \in[1,7]$. Note that in this case, although unknown to the reconstruction process, the nature of the problem is different to that given by the two test functions (4) and (5) given in Section III. Instead, the problem here can be regarded as a combination of problems represented by both (4) and (5). This is because the global optimum for this reconstruction problem is a vector of dimension 27, where only one of its elements is located close to one end of the boundary while the rest of the elements lie on the other end of the boundary of the search 
space.

In the implementation of PSO, we have tested all four boundary conditions with the same initial population, and for each boundary condition 10 simulations have been performed, each with 500 iterations, to compute the average fitness value versus the number of iterations.

It is also worth mentioning here that in the absence of the measured data, we have synthesized our measured scattered field values using the FDTD technique, and used MoM with volume integral formulation for the forward problem. We have avoided committing the "inverse crime" by not using the same numerical technique in the inversion algorithm as is used for synthesizing the measured data of the scattered field [10]. Furthermore, we have also considered the effect of random noise on the measured data by taking the signal-to-noise ratio to be equal to $20 \mathrm{~dB}$.

The results plotted in Fig. 4 show the average fitness values obtained over 500 iterations when all four boundary conditions are used. As expected, for this reconstruction problem, the performance of the absorbing boundary was superior since 26 out of 27 optimum values lie on the boundary of the search space $\left(\varepsilon_{r}=1\right)$. However, as indicated in the results presented in Section III, the superiority offered by the absorbing boundary may not always be guaranteed in other cases. A typical example is shown in Fig. 2(d), where the absorbing boundary performs the best for the type II case but performs the worst for type I case. Hence, it would cause great uncertainty in deciding as to which boundary condition be appropriate for a given optimization problem. Wrong choice of an inefficient boundary condition for a given problem such as the microwave image reconstruction could result in poor reconstruction accuracy in addition to waste of huge computational resources. On the other hand, the performance of the proposed damping boundary has always performed consistently well in all cases, and Fig. 4 further testifies the robustness it can offer. From Fig. 4, it is also evident that the proposed damping boundary has outperformed both the invisible and reflecting boundaries. Thus, the use of the proposed damping boundary has eliminated the uncertainty arising from the choice of inappropriate boundary condition for PSO. The results in Fig. 5 show a comparison of the final images (averaged after 10 simulation runs) obtained from PSO runs utilizing each 
of the four boundary conditions. It is quite clear from Fig. 5 that the damping boundary has successfully reconstructed the value of $\varepsilon_{r}$ for the dielectric scatterer, and outperformed some of the other boundary conditions, while offering robustness and consistency.

\section{CONCLUSION}

In this paper, a simple hybrid boundary condition that features a damping characteristic has been proposed for PSO technique. The results have shown that the proposed damping boundary can provide a much robust and consistent optimization performance as compared to the existing boundary conditions regardless of the dimensionality of the problem as well as the location of the global optimum with respect to the search space boundary. Thus, the proposed damping condition would strengthen the PSO to be more effective in the optimization of many other difficult and complex engineering problems such as microwave image reconstruction.

\section{REFERENCES}

[1] R. C. Eberhart and J. Kennedy, "A new optimizer using particle swarm theory," in Proc. $6^{\text {th }}$ Symp. Micro Machine and Human Science, Nagoya, Japan, 1995, pp. 39-43.

[2] _ _ "Particle swarm optimization," in Proc. IEEE Conf. Neural Networks IV, Piscataway, NJ, 1995, pp. 19421948.

[3] J. Robinson and Y. Rahmat-Samii, "Particle swarm optimization in electromagnetics," IEEE Trans. Antennas Propagat., vol. 52, pp. 397-407, Feb. 2004.

[4] F. van den Bergh and A. P. Engelbrecht, "A cooperative approach to particle swarm optimization," IEEE Trans. Evol. Comput., vol. 8, pp. 225-239, Jun. 2004.

[5] S. Caorsi, G. L. Gragnani, and M. Pastorino, "Redundant electromagnetic data for microwave imaging of threedimensional dielectric objects," IEEE Trans. Antennas Propagat., vol. 42, pp. 581-589, 1994.

[6] Y. Shi and R. C. Eberhart, "Parameter selection in particle swarm optimization," in Evolutionary Programming, V. W. Porto, N. Saravanan, D. Waagen, and A. E. Eiben, Eds. Berlin, Germany: Springer-Verlag, 1998, vol. VII, pp. 591-600. 
[7] —_ "A modified particle swarm optimizer," in Proc. IEEE Conf. Evolutionary Computation, Anchorage, AK, 1998, pp. 69-73.

[8] — - "Particle swarm optimization: Developments, applications and resources," in Proc. 2001 Congress on Evolutionary Computation, Seoul, Korea, 2001, pp. 81-86.

[9] M. Clerc and J. Kennedy, "The particle swarm-explosion, stability, and convergence in a multidimensional complex space," IEEE Trans. Evol. Comput., vol. 6, pp. 58-73, Feb. 2002.

[10] P. M. van den Berg, M. G. Cote, and R. E. Kleinman, "Blind shape reconstruction from experimental data," IEEE Trans. Antennas Propagat., vol. 43, pp. 1389-1396, Dec. 1995.

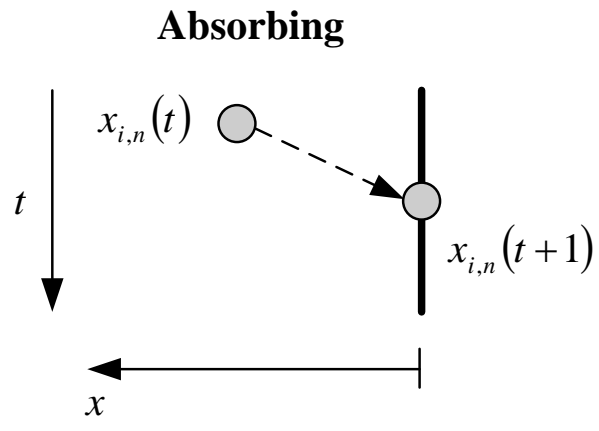

(a)

Invisible

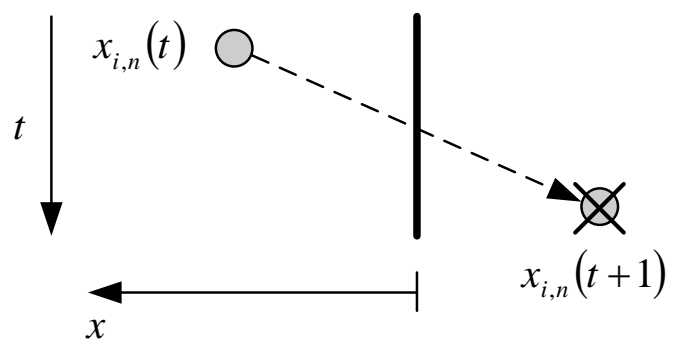

(c)
Reflecting

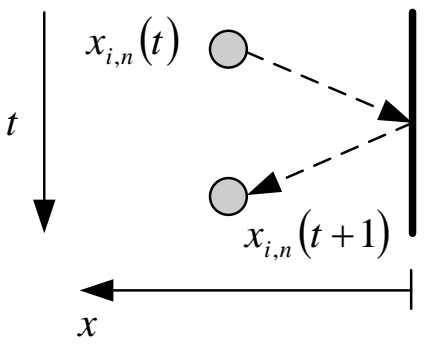

(b)

\section{Damping}

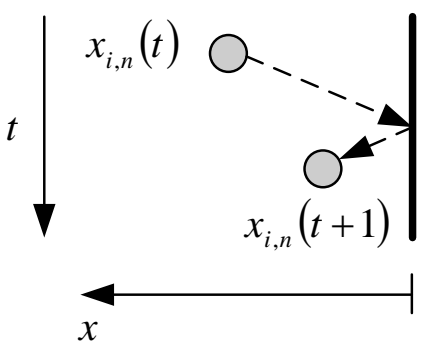

(d)

Fig. 1. (a) For absorbing boundaries the velocity of the particle is zeroed and the particle is stopped at the boundary. (b) For reflecting boundaries the sign of the velocity is reversed and the particle is reflected back to the search space after the impact. (c) For invisible boundaries the particle is allowed to escape the boundary of the search space and is ignored by the fitness evaluator. (d) For damping boundaries part of the velocity is absorbed by the boundary during the impact and the particle is then reflected back with a lesser velocity of a reversal of sign. 

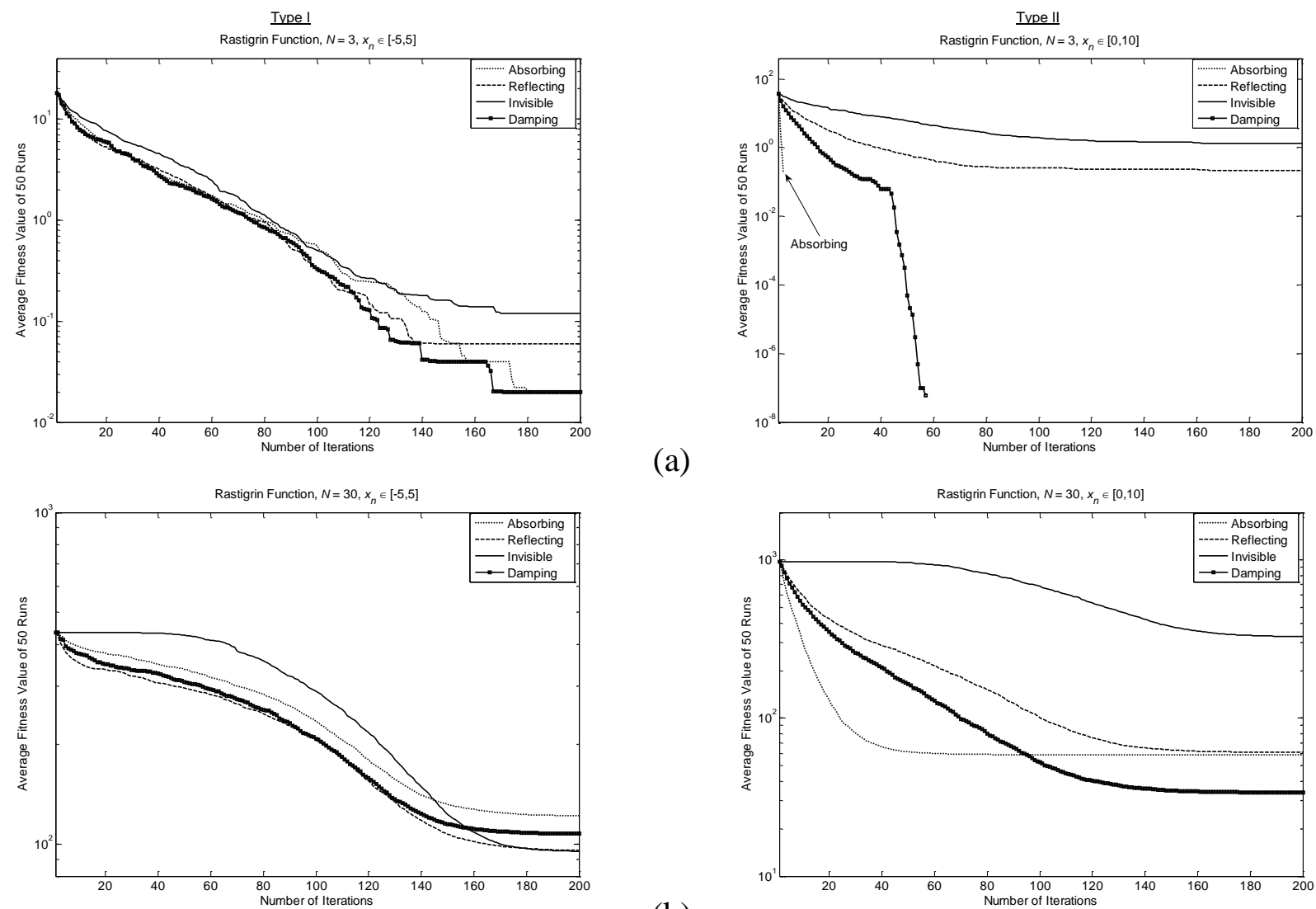

(a)
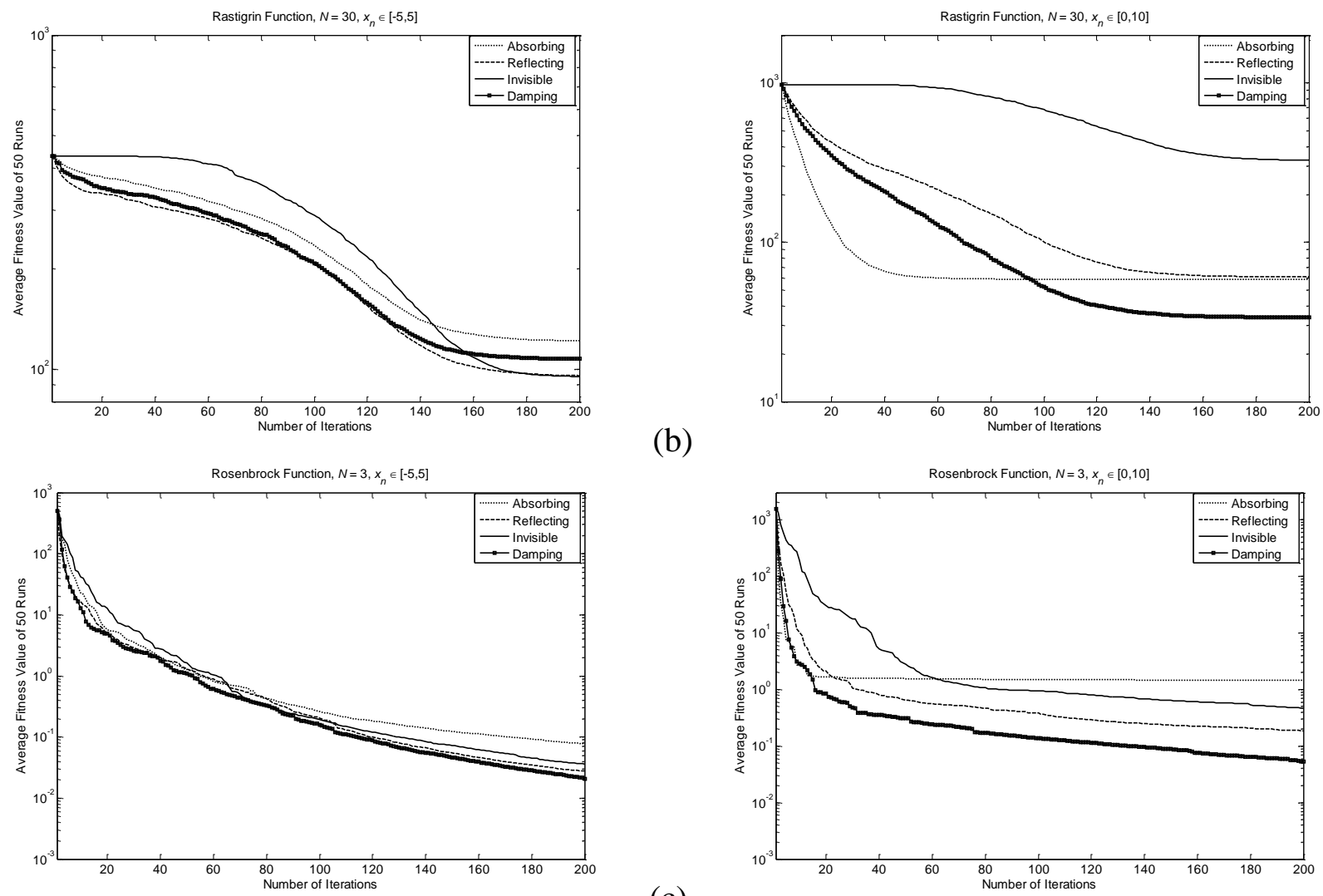

(b)
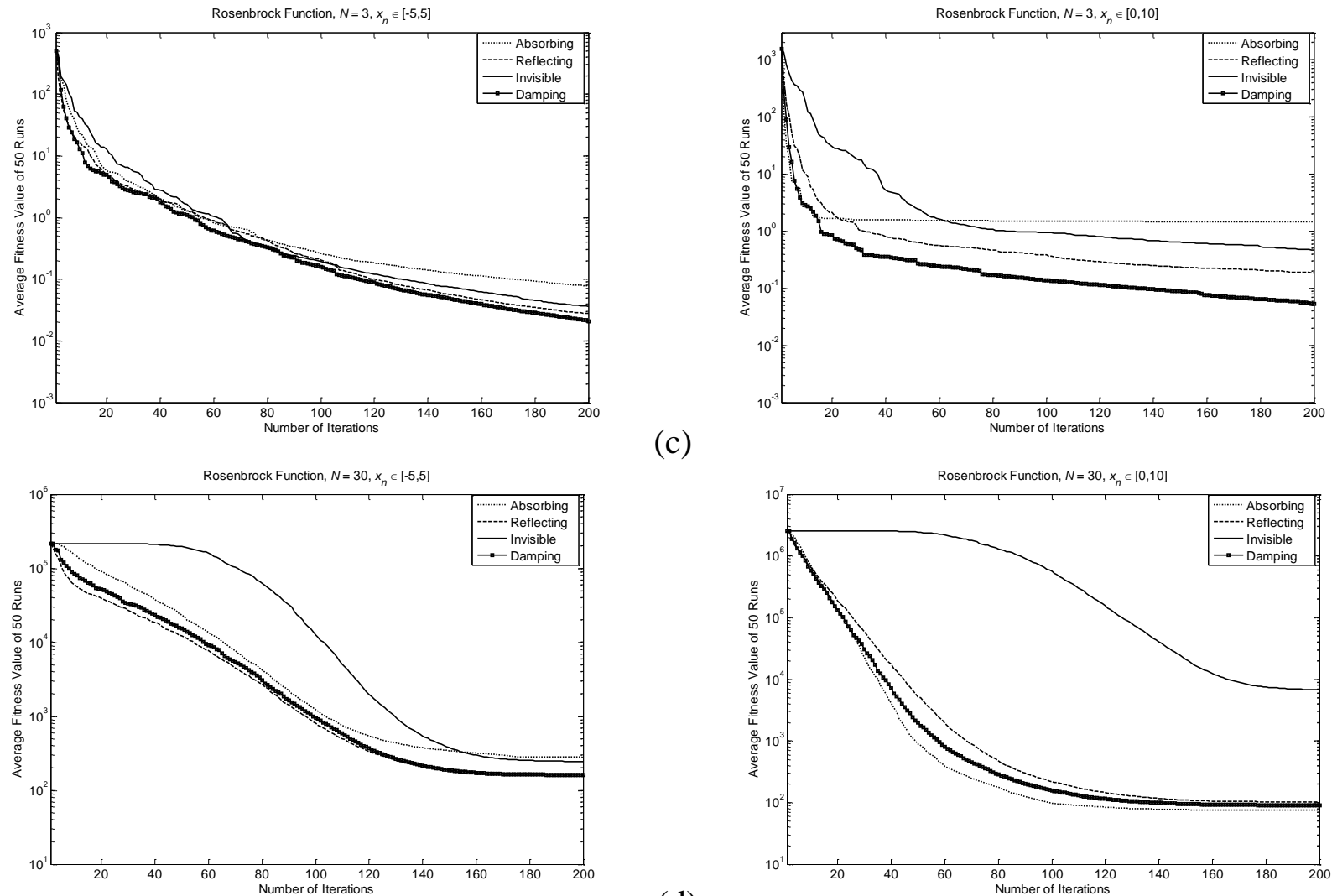

(d)

Fig. 2. The average fitness value obtained versus the number of iterations for the 50 independent PSO runs of (a) 3-D Rastigrin function. (b) 30-D Rastigrin function. (c) 3-D Rosenbrock function. (d) 30-D Rosenbrock function. 


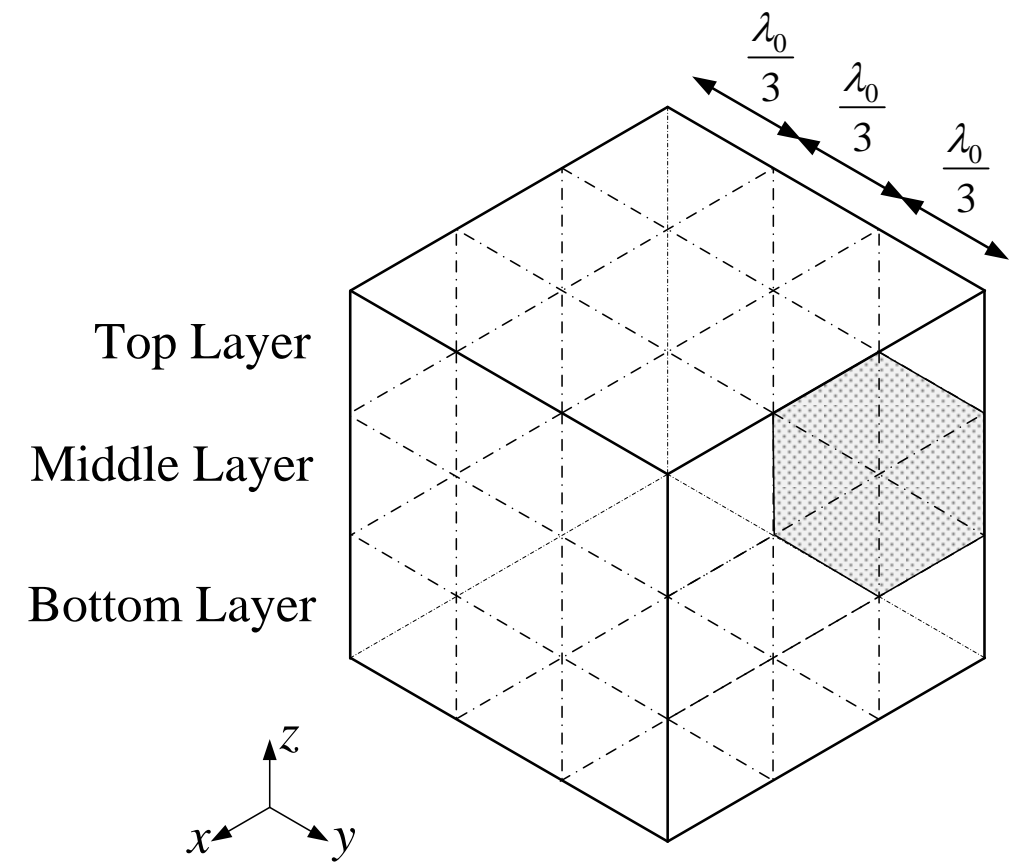

Fig. 3. The problem geometry of the microwave imaging example. The dielectric scatterer is denoted by the shaded area in the investigation domain.

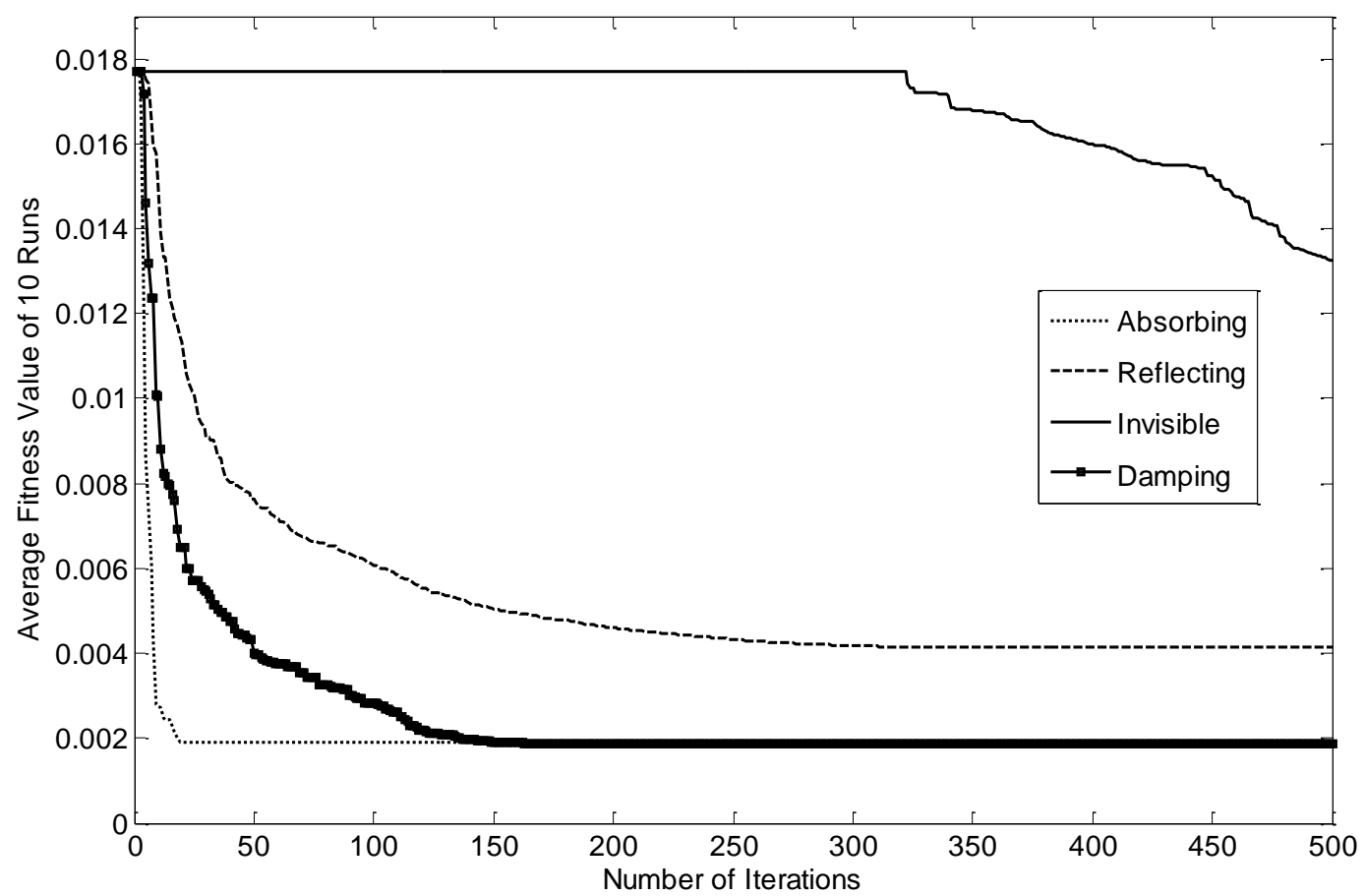

Fig. 4. The average fitness value obtained versus the number of iterations for the 10 independent PSO runs of the microwave image reconstruction example. 

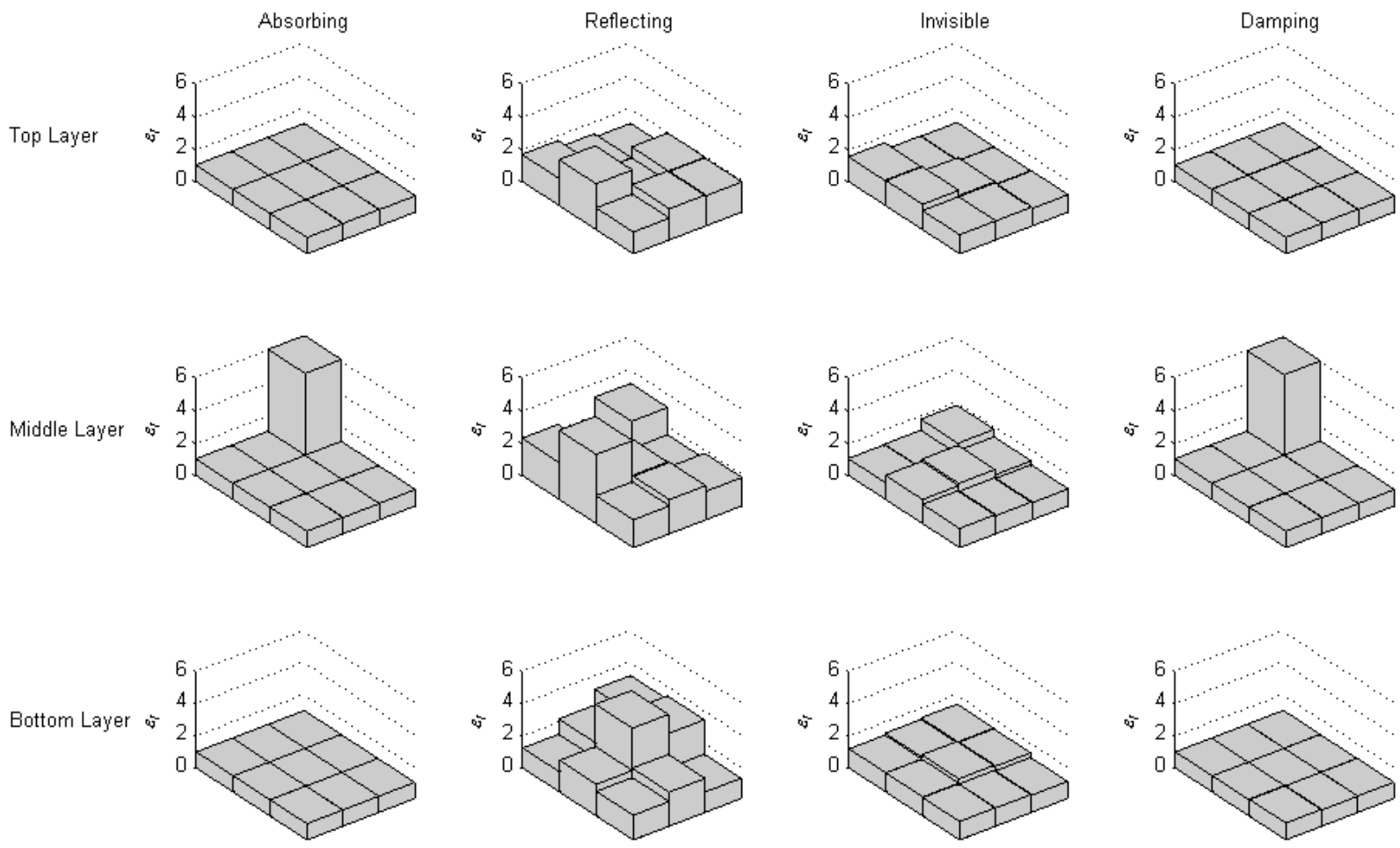

Fig. 5. The reconstructed images that show the distribution of $\varepsilon_{r}$ for the top, middle, and bottom layer of the investigation domain. 